\title{
A Study on Chronofatality Trends of COVID-19 Deaths at a Tertiary Care Hospital
}

\author{
Kattamreddy Ananth Rupesh', Mopuri Venkateswarlu', Suresh Pamujula', \\ Pravin Panditrao Kalyankar², Nitesh Motiram Salvi², and Shayali Vora ${ }^{3}$ \\ 'Department of Forensic Medicine, A.C. Subba Reddy Government Medical College (ACSR GMC), Nellore, Andhra Pradesh, India \\ ${ }^{2}$ Occupational Health Services, Hindustan Petroleum Corporation Limited. (HPCL), Mumbai, Maharashtra, India \\ ${ }^{3}$ Health Care Administration, Canadore College, North Bay, Canada
}

The aim of the study is to find out any association between time of death in coronavirus disease (COVID-19) patients and variables like age, sex, and existence of comorbidities including type II diabetes mellitus, hypertension, coronary artery disease, chronic kidney disease, etc. An attempt was also made to elucidate the reasons for relationship between time of death and other aforementioned variables. Mortality data of 1,553 COVID-19 cases from a tertiary care hospital between March 2020 to September 2021 were analyzed. Maximum deaths occurred between 18:01 hours to $06: 00$ hours of the 24-hour cycle. There is a significant statistical association between time of death and age, time of death and sex, time of death and having a comorbidity of diabetes mellitus in the study sample. The study confirms that the chronofatality of COVID-19 deaths has a nocturnal predilection. The circadian rhythms of glucocorticoids, respiratory physiology of sleep, and circadian hemodynamic variations may have a role in prognosis and fatality of COVID-19.

Keywords: Chronobiology phenomenon; Circadian clock; COVID-19; Chronotherapy; SARS-CoV-2

Received: October 2, 2021 Revised: November 1, 2021 Accepted: November 12, 2021

Corresponding author: Kattamreddy Ananth Rupesh, MD, Department of Forensic Medicine, ACSR Government Medical College, Nellore, Andhra Pradesh 524004 , India.

Tel: 91-82977-16897, E-mail: ananth.kattam@gmail.com

@ This is an Open Access article distributed under the terms of the Creative Commons Attribution Non-Commercial License (https://creativecommons.org/licenses/by$\mathrm{nc/4.0)}$ which permits unrestricted non-commercial use, distribution, and reproduction in any medium, provided the original work is properly cited.

\section{INTRODUCTION}

The science of chronomedicine explores the interaction between biological rhythms, medicine and drugs [1]. Circadian rhythms operate at the cellular, tissue, and organismal level in humans. In general, there is a synchrony between the central suprachiasmatic nucleus (SCN) and the other organ clocks in human body. At the molecular level, positive (CLOCK/NPAS2, BMAL1, RORs) and negative (PERIODs, CRYPTOCHROMEs, REV-ERBs) genes influence time bound events [2].

The importance of circadian rhythms in integrating and regulating biological systems is well established. Melatonin is a chronomodulation factor in several metabolic processes comprising glucose homeostasis of human and has been experimented in management of type II diabetes [3]. It is well known that, derangement of chrono-rhythms can potentiate the onset of several diseases. For instance, there is a link between Parkinson's disease and circadian clock disruption [4]. Disturbed chrono-rhythms increase cardiac disease risks and outcomes [5]. Additionally, mood disorders are associated with disrupted circadian clock-controlled responses, such as sleep and cortisol secretion. Similarly, myocardial infarction and acute ischemic strokes are disproportionately high during the morning hours [6]. Likewise, nocturnal asthma represents the exaggerated amplitude of normal circadian patterns because of increased airway responsiveness and decreased lung function at night. The crux of chrono-therapy delivers drugs at an appropriate time, and the use of statins as a single dose at night is a classic case [6-9].

In this study, we endeavor to comprehensively analyze coronavirus disease (COVID-19) mortality data from the perspective of circadian medicine.

\section{METHODS}

This is a cross-sectional observational study. COVID-19 mortality data available from death summaries of COVID-19 cases 
reported at Government General Hospital, Nellore was reviewed. The duration of the study was from March 2020 to September 2021. All available COVID-19 patient deaths reported at the hospital during the study period were taken as convenient sample and included in the study.

Age, sex, presence or absence of comorbidities like hypertension, diabetes, coronary artery disease, date and time of death, and other relevant data were extracted from the death summaries. Deaths clustered in each hour of the day and every six hours were grouped for studying. Mere presence or absence of comorbidities like hypertension, type II diabetes, chronic kidney disease and coronary artery disease were considered.

All the parameters were analyzed with the time of death to check whether any association existed between them using Pearson Chi Square test, likelihood ratio and linear by linear association wherever applicable. Data was analyzed using SPSS Software version 28 (IBM Corp., Armonk, NY, USA). Results were considered at a nominal significance of $p<0.05$. An approval of institutional ethics committee was obtained to conduct the study. The institutional ethics committee has waived the requirement to obtain an informed consent from the patients as the study involved only blinded mortality data.

\section{RESULTS}

Mortality data of a total of 1,553 deaths was analyzed. The actual number of COVID-19 deaths at the institution may be up to $5 \%$ higher than reported in the study. Among the 1,553 deaths, $62.8 \%$ were male and $37.2 \%$ were female. The mean age of COVID-19 deaths is $57.78 \pm 13.74$ years for the whole data analyzed. The maximum number of deaths, accounting for $6.8 \%$ of all deaths, occurred between 00:01 hours to 01:00 hours in a 24-hour clock cycle. The least number of deaths, i.e., $1.8 \%$, occurred between 08:01 hours to 09:00 hours of the 24-hour cycle. Maximum deaths (a total of 404) were reported in May 2021 during the entire course of the first and second waves of the pandemic. The second wave of the pandemic was indeed very severe and cases peaked quickly in May 2021.

The maximum deaths were reported between 18:01 hours to 06:00 hours of the day. A total of 411 deaths occurred between 18:01 hours to 24:00 hours and 407 deaths occurred between 00:01 hours to 06:00 hours. Less number of deaths was recorded during the first half of the day, i.e., 358 deaths, between 06:01 hours to 12:00 hours (Figure 1).

There is a significant statistical association between the time of death and the age of the deceased $(\mathrm{p}<0.001)$. There is also a significant statistical association between the time of death and having comorbidity of diabetes mellitus $(\mathrm{p}<0.004)$. Furthermore, there is a significant statistical association between the time of death and the sex of the deceased $(\mathrm{p}<0.007)$.

There is no statistical association between time of death and comorbidity of hypertension; time of death and comorbidity of coronary artery disease; time of death and comorbidity of chron-

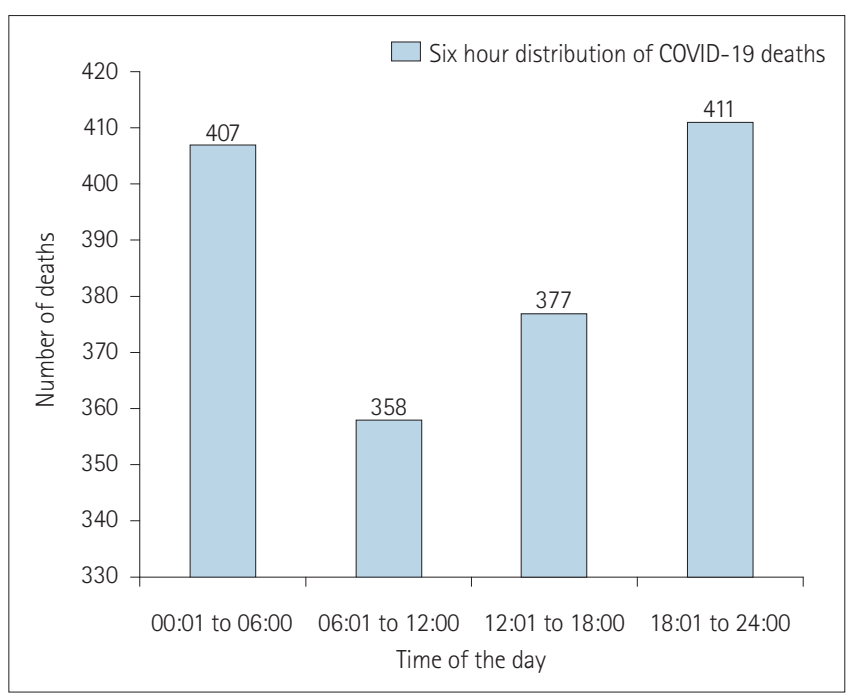

Figure 1. Distribution of COVID-19 deaths in each quarter of the day.

ic kidney disease. Hypothyroidism and cerebrovascular accident were seen as comorbidities in a few cases and hence they were not analyzed.

\section{DISCUSSION}

Close to $85 \%-90 \%$ of all the deaths reported in this study were from COVID-19 ICU, wherein all forms of non-invasive ventilation were used. It is also necessary to state that invasive ventilation and cardiopulmonary resuscitation were not carried out in majority of the cases considering high risk of aerosol spread and less chance of reviving them. Midazolam was used for the purpose of analog-sedation in COVID-19 patients. Methyl prednisolone was administered to majority of patients admitted in ICU twice daily. In addition, low molecular weight heparin was used wherever it was necessary. The protocols for management of COVID-19 ICU cases were uniform as there were a huge number of admissions during the peak season. Therefore, the treatment related variables were not factored in the study.

COVID-19 results in endothelial inflammation, intercellular junctional damage, and micro-thrombi formation. Hence, chronorythyms of blood circulation may influence vascular thromboembolic phenomena in COVID-19 cases [10]. Physiological processes like immune response, oxidative stress, and cellular metabolism are also known to be regulated by the circadian clock $[11,12]$. By the same token, the behavior of asymptomatic carriers, organ physiology, and sleep pattern in the sick and the viral life cycle within infected cells are linked to circadian clock in COVID-19 patients [13,14].

Additionally, COVID-19 induces myocardial injury, arrhythmia, acute coronary syndrome, venous thromboembolism and also worsens the existing cardiac disease [15]. Besides, the biological clock plays important role in the pathogenesis of severe acute respiratory syndrome coronavirus 2 (SARS-CoV-2) infection mediated through ACE2 and BMAL1 [16]. 
The relationship between cause of death and the time of death in critically ill COVID-19 patients was studied by few researchers [17]. Though circadian trends of infection were researched till now [18], no studies were done so far in understanding the significance of the exact time of death from a circadian rhythm perspective. Hence, the present study is justified.

In this study, it is observed that there is a nocturnal predilection in fatality of COVID-19 cases during both waves of the pandemic. The reasons for the majority of deaths happening in the nights are to be investigated from the pathophysiological angle. SARSCoV-2 infection is affected by the patient's circadian clock system through two major mechanisms 1) by direct viral replication within the host's cells and 2) by indirect effects on innate and adaptive immune responses. The total viral load and viral clearance in the host are influenced by circadian clocks. The BMAL1 gene has a role in mouse herpes virus infection and similar associations with SARS-CoV-2 infection need to be need to be thoroughly investigated.

The renin-angiotensin mechanism and ACE2 receptor regulation which is critical in COVID-19 pathophysiology is regulated by circadian rhythms [16]. Correspondingly, hypoxemic respiratory failure is one of the major causes of death in COVID-19 cases. The role of hypoxia-inducible factor 1 alpha being an important interlocutor between circadian clocks and cellular oxygenation is one more arena to be further studied.

The endogenous secretion of glucocorticoids has a robust circadian oscillation with a daily peak around the time of sleep-wake transition and minimal levels in the evening and early part of the night [19]. Low biological cortisol levels late in the evenings and into the nights perhaps can be one reason leading to a pro-inflammatory milieu resulting in the nocturnal preference of COVID-19 deaths. Though the present study was carried out at one particular center, several personal communications to authors from physicians involved in COVID-19 care at several centers also confirmed high nocturnal propensity of COVID-19 fatalities. This needs to be validated by multi-centric studies.

The physiology of sleep can also influence COVID-19 fatalities. Regulation of sleep differs significantly between sleep and wakefulness; ventilatory responses to oxygen and carbon dioxide differ in sleep in comparison to wakefulness. Response to airway resistance also varies during sleep and there is a significant reduction in chemosensitivity to oxygen and carbon dioxide with the onset of sleep [20]. Hence, it can be assumed that the reduced breathing effort of an individual combined with the nocturnal physiology of respiration can accentuate inflammatory changes in the lungs apart from the natural course of the illness.

SARS-CoV-2 perturbation of host circadian rhythms and the host response to the virus leading to cytokine storm is clock-regulated [21]. A circadian disruption leading to hyper inflammatory state is being counteracted with melatonin adjunctive therapy [22].

Circadian rhythms can influence the pathology of arteriosclerosis and thrombosis [23]. It is known that many thromboembolic events occur during the early hours of the day because of a high proclivity for platelet aggregation and vasoconstriction during the early morning hours. This could also be one reason for high early morning deaths in COVID-19 cases where non-respiratory causes of death predominate. The association between time of death and diabetes mellitus is quite intriguing and requires further research to propose a hypothesis.

Patient compliance factors, severity and duration of comorbidities were not taken into account in the study. Hence, subsequent researchers are advised to do so.

Chronofatality studies are new in the field of circadian medicine. Understanding the significance of circadian rhythms in the timing of death will provide new insights into pathophysiology. The study demonstrated nocturnal predisposition of COVID19-related deaths in hospital settings. The existence of an association between time of death and variables like age, sex, and having a comorbidity of diabetes mellitus will guide future researchers working in this area of chronobiology in medicine.

\section{Acknowledgments}

We thank Mr. Ramesh Chandra Menda, Mr. Kumar Nakka, and Mr. Alla Penchal Kumar for assisting in data acquisition and digitalization. We express our heartfelt gratitude to Dr. N. Khader Vali and Dr. Sasikala P, for their support in analyzing the data. Inputs from Dr. Namdev V and Dr. Krishna S helped formulate the discussion part of the article.

\section{Conflicts of Interest}

The authors have no potential conflicts of interest to disclose.

\section{Author Contributions}

Conceptualization: Kattamreddy Ananth Rupesh. Data curation: all authors. Formal analysis: Kattamreddy Ananth Rupesh. Investigation: all authors. Methodology: Kattamreddy Ananth Rupesh. Project administration: Kattamreddy Ananth Rupesh. Resources: all authors. Software: Kattamreddy Ananth Rupesh. Supervision: Kattamreddy Ananth Rupesh. Validation: all authors. Visualization: all authors. Writing_original draft: Kattamreddy Ananth Rupesh. Writing—review \& editing: all authors.

\section{ORCID iDs}

Kattamreddy Ananth Rupesh (1)

https://orcid.org/0000-0003-1525-3740

Mopuri Venkateswarlu (1)

https://orcid.org/0000-0002-4612-3650

Suresh Pamujula (1)

https://orcid.org/0000-0002-1134-332X

Pravin Panditrao Kalyankar (16

https://orcid.org/0000-0002-6187-8138

Nitesh Motiram Salvi (1)

https://orcid.org/0000-0002-6930-7655

Shayali Vora (1)

https://orcid.org/0000-0003-0652-4895 


\section{REFERENCES}

1. Maheshwari A, Verma N. Chronomedicine: a unique concept to manage human diseases (chapter 75). In: Muruganathan A, editor. Progress In Medicine 2017. Mumbai: Association of Physicians of India, 2017, p. 415-418.

2. Chen Z. What's next for chronobiology and drug discovery. Expert Opin Drug Discov 2017;12:1181-1185.

3. Forrestel AC, Miedlich SU, Yurcheshen M, Wittlin SD, Sellix MT. Chronomedicine and type 2 diabetes: shining some light on melatonin. Diabetologia 2017;60:808-822.

4. De Lazzari F, Bisaglia M, Zordan MA, Sandrelli F. Circadian rhythm abnormalities in parkinson's disease from humans to flies and back. Int J Mol Sci 2018;19:3911.

5. Khaper N, Bailey CDC, Ghugre NR, Reitz C, Awosanmi Z, Waines R, et al. Implications of disturbances in circadian rhythms for cardiovascular health: a new frontier in free radical biology. Free Radic Biol Med 2018;119:85-92.

6. Elliott WJ. Circadian variation in the timing of stroke onset: a meta-analysis. Stroke 1998;29:992-996.

7. Adam D. Core concept: emerging science of chronotherapy offers big opportunities to optimize drug delivery. Proc Natl Acad Sci U S A 2019;116:2195721959.

8. Walker WH 2nd, Walton JC, DeVries AC, Nelson RJ. Circadian rhythm disruption and mental health. Transl Psychiatry 2020;10:28.

9. Martin RJ. Nocturnal asthma: circadian rhythms and therapeutic interventions. Am Rev Respir Dis 1993;147(6 Pt 2):S25-S28.

10. Loo J, Spittle DA, Newnham M. COVID-19, immunothrombosis and venous thromboembolism: biological mechanisms. Thorax 2021;76:412-420.

11. Xie Y, Tang Q, Chen G, Xie M, Yu S, Zhao J, et al. New insights into the circadian rhythm and its related diseases. Front Physiol 2019;10:682.

12. Giri A, Srinivasan A, Sundar IK. COVID-19: sleep, circadian rhythms and immunity - repurposing drugs and chronotherapeutics for SARS-CoV-2. Front Neurosci 2021;15:674204.

13. Meira E Cruz M, Miyazawa M, Gozal D. Putative contributions of circadian clock and sleep in the context of SARS-CoV-2 infection. Eur Respir J 2020; 55:2001023.

14. Haspel J, Kim M, Zee P, Schwarzmeier T, Montagnese S, Panda S, et al. A timely call to arms: COVID-19, the circadian clock, and critical care. J Biol Rhythms 2021;36:55-70.

15. Nishiga M, Wang DW, Han Y, Lewis DB, Wu JC. COVID-19 and cardiovascular disease: from basic mechanisms to clinical perspectives. Nat Rev Cardiol 2020;17:543-558.

16. Tomar R, Raghav A. Association of circadian clock and severe acute respiratory syndrome coronavirus 2 infection. Chronobiol Med 2021;3:60-63.

17. Contou D, Cally R, Sarfati F, Desaint P, Fraissé M, Plantefève G. Causes and timing of death in critically ill COVID-19 patients. Crit Care 2021;25:79.

18. Ray S, Reddy AB. COVID-19 management in light of the circadian clock. Nat Rev Mol Cell Biol 2020;21:494-495.

19. Oster H, Challet E, Ott V, Arvat E, de Kloet ER, Dijk DJ, et al. The functional and clinical significance of the 24-hour rhythm of circulating glucocorticoids. Endocr Rev 2017;38:3-45.

20. Newton K, Malik V, Lee-Chiong T. Sleep and breathing. Clin Chest Med 2014; 35:451-456.

21. Sengupta S, Ince L, Sartor F, Borrmann H, Zhuang X, Naik A, et al. Clocks, viruses, and immunity: lessons for the COVID-19 pandemic. J Biol Rhythms 2021;36:23-34.

22. Zhang R, Wang X, Ni L, Di X, Ma B, Niu S, et al. COVID-19: melatonin as a potential adjuvant treatment. Life Sci 2020;250:117583.

23. Man AWC, Li H, Xia N. Circadian rhythm: potential therapeutic target for atherosclerosis and thrombosis. Int J Mol Sci 2021;22:676. 